\title{
Exploration of the Trade Space Between Unmanned Aircraft Systems Descent Maneuver Performance and Sense-and-Avoid System Performance Requirements
}

\author{
Devin P. Jack ${ }^{1}$, Keith D. Hoffler ${ }^{2}$, and Sally C. Johnson ${ }^{3}$ \\ Adaptive Aerospace Group, Inc., Hampton, VA 23666
}

\begin{abstract}
A need exists to safely integrate Unmanned Aircraft Systems (UAS) into the United States' National Airspace System. Replacing manned aircraft's see-and-avoid capability in the absence of an onboard pilot is one of the key challenges associated with safe integration. Sense-and-avoid (SAA) systems will have to achieve yet-to-be-determined required separation distances for a wide range of encounters. They will also need to account for the maneuver performance of the UAS they are paired with. The work described in this paper is aimed at developing an understanding of the trade space between UAS maneuver performance and SAA system performance requirements, focusing on a descent avoidance maneuver. An assessment of current manned and unmanned aircraft performance was used to establish potential UAS performance test matrix bounds. Then, near-term UAS integration work was used to narrow down the scope. A simulator was developed with sufficient fidelity to assess SAA system performance requirements. The simulator generates closest-point-of-approach (CPA) data from the wide range of UAS performance models maneuvering against a single intruder with various encounter geometries. Initial attempts to model the results made it clear that developing maneuver performance groups is required. Discussion of the performance groups developed and how to know in which group an aircraft belongs for a given flight condition and encounter is included. The groups are airplane, flight condition, and encounter specific, rather than airplane-only specific. Results and methodology for developing UAS maneuver performance requirements are presented for a descent avoidance maneuver. Results for the descent maneuver indicate that a minimum specific excess power magnitude can assure a minimum CPA for a given time-togo prediction. However, smaller amounts of specific excess power may achieve or exceed the same CPA if the UAS has sufficient speed to trade for altitude. The results of this study will support UAS maneuver performance requirements development for integrating UAS in the NAS. The methods described are being used to help RTCA Special Committee 228 develop requirements.
\end{abstract}

\section{Nomenclature}

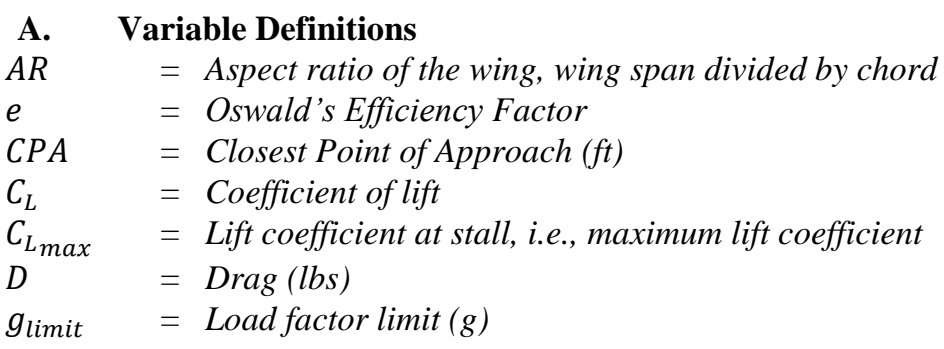

\footnotetext{
${ }^{1}$ AIAA Member, Research and Development Engineer.

2 AIAA Associate Fellow, President/Senior Research and Development Engineer.

${ }^{3}$ Senior Research and Development Engineer.
} 

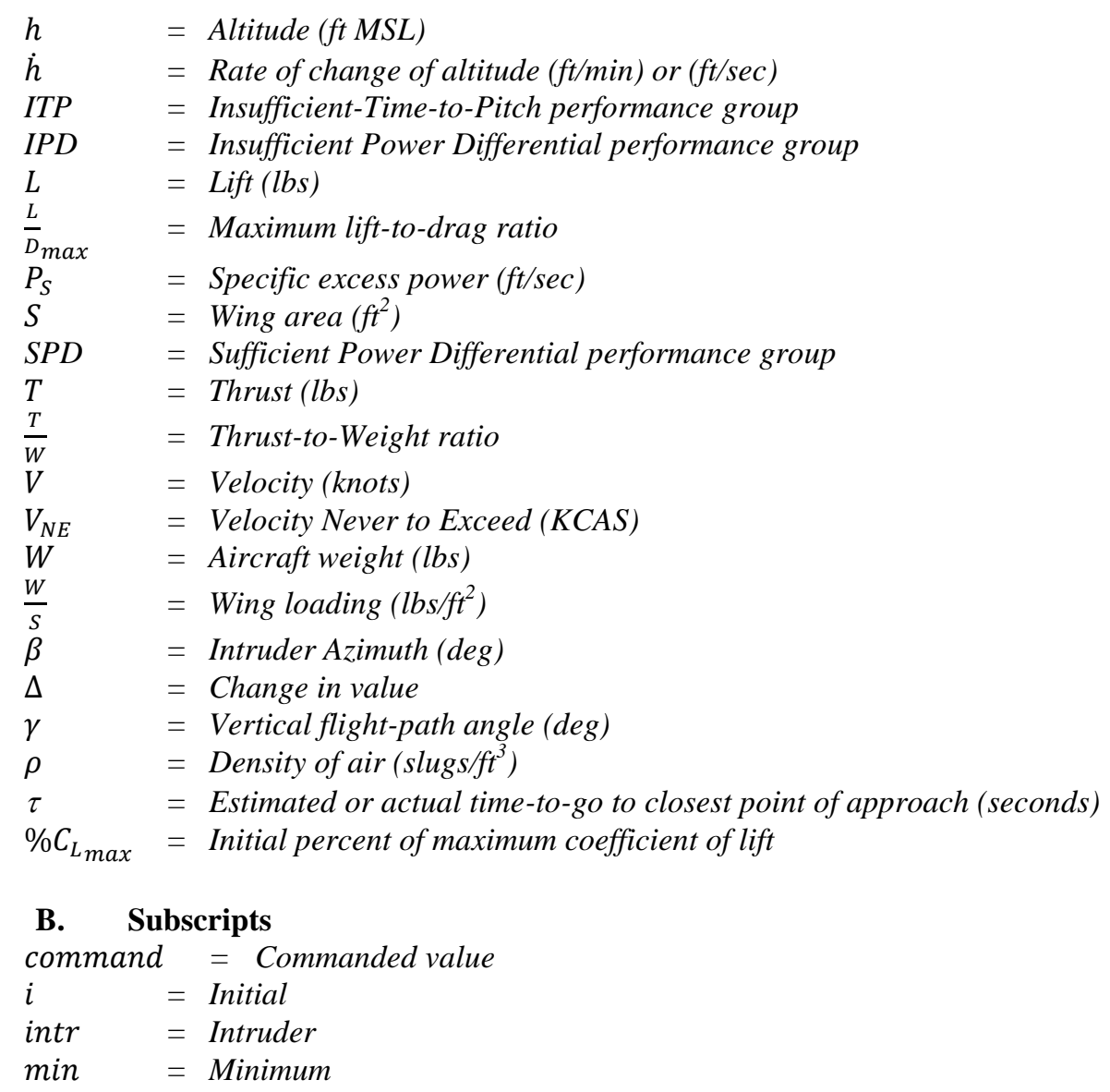

\section{Introduction}

$\mathrm{T}$ he United States National Airspace System (NAS) has evolved to both support and rely on the capabilities of manned aircraft. Currently, the Department of Defense (DOD), other public entities, and private industry are showing increasing interest in gaining regular access to fly unmanned aircraft systems (UAS) in the NAS. One of the major barriers to integrating UAS in the NAS is the requirement to see and avoid other aircraft, a task currently performed by the onboard pilot. Per Title 14 of the Code of Federal Regulations (14 CFR) Part 91.113 (Ref. 1), "vigilance shall be maintained by each person operating an aircraft so as to see and avoid other aircraft." Thus, pilots are expected to see and avoid other aircraft and to maneuver in predictable ways to preserve the safety, orderliness, and efficiency of the NAS environment. UAS will be expected to operate in a similar manner, but with sense and avoid (SAA) systems replacing the see-and-avoid capability of manned aircraft. To date, the acceptable design space and required capabilities for SAA in this environment remains largely undefined. A large body of work related to collision avoidance was generated during the development of the Traffic Alert and Collision Avoidance System (TCAS) (Ref. 3). However, TCAS was generally designed and intended for use on piloted transport-category airplanes and requires higher climb and descent performance than the broad array of current manned aircraft exhibit. The widely diverse UAS fleet often exhibits even less maneuver performance currently, sometimes because of airframe and power-plant limitations and sometimes because of the flight-control system.

To analyze the interaction of UAS and SAA system performance requirements specific to separation assurance for UAS, a simplified simulation tool was developed and a simulation experiment was conducted. For each simulation run, a UAS and a non-maneuvering intruder are set on a collision course, and the UAS immediately initiates a maneuver to avoid the intruder, with resulting Closest Point of Approach (CPA) and other airplane states recorded. The current paper details vertical descent avoidance maneuvers evaluated across a range of descent rates with airplane performance limitations impacting the resulting maneuver. A previous analysis of commanded climb and level-turn maneuvers is documented in Ref. 4. The reference also includes a more in depth description of the 
simulator, models, and approach. It includes some descent maneuver results as well. This paper expands on those results.

The simulation tool allows broad and rapid run-to-run variation of UAS design parameters that impact maneuver performance. Design parameter ranges used for this study covered a broad range of present and future UAS aerodynamic and propulsive performance and maneuverability, but focused on performance ranges that could potentially limit conduct of avoidance maneuvers. Encounters studied were based on near-term UAS integration concepts as defined in the RTCA Special Committee - Minimum Performance Standards for Unmanned Aircraft Systems (SC-228) Terms of Reference (Ref. 5) and work plan (Ref. 6). Even with the parameter range reductions, millions of combinations of airplane performance and encounter parameters were analyzed in an effort to describe the trade space between SAA system and UAS maneuver performance requirements. Separate simulation models were developed to account for UAS maneuver performance limitations associated with climb, descent, and levelturn maneuvers independently, which significantly reduced model complexity and, therefore, the amount of time required for each simulation run. Before data gathering began, a verification and validation study was performed to ensure that the simulation accurately modeled maneuver performance across the parameter ranges of interest.

The encounter scenarios were designed to result in CPAs spanning from near mid-air-collision (NMAC) ranges to well beyond those that could be considered "well clear." Since "well clear" has yet to be quantitatively defined, encounters leading to a wide range of CPAs were included in the study, with vertical CPA distances from less than $100 \mathrm{ft}$ to greater than $1000 \mathrm{ft}$.

Using results from the simulation, a number of candidate performance parameters were evaluated to determine the best universal predictors of CPA. The objective was to identify a set of performance parameters that could be used to predict the avoidance maneuver result given current or future UAS design parameters. In order to develop effective performance predictors, maneuver performance groups were identified and analyzed separately, i.e., whether and how the UAS was able to achieve and sustain the commanded descent maneuver for each encounter case. The maneuver performance groups are dependent on airplane configuration, initial flight condition, and encounter parameters, not airplane alone. The groups are described herein.

The results and methodology presented in this paper will help regulators determine minimum maneuver performance requirements for UAS operating in the NAS with a SAA system. Results will also help determine the point in the SAA system timeline at which the maneuver must be initiated, whether the desired maneuver is maximum performance or something less. The resulting trade space is expected to support development of flexible SAA system design requirements, thus accommodating as many UAS types in the NAS as possible.

\section{Simulation Tool and Airplane Performance Models}

A simulation tool was developed to evaluate SAA maneuver time requirements for a spanning set of airplane performance models over a broad range of encounters. The airplane models are defined by basic design parameters, thus the results are generally applicable rather than tied to specific airplanes, and a spanning set of performance capabilities can be evaluated by varying the design parameters across a range of values of interest. The tool, which is described in more detail in Ref. 4, is referred to as the 2-degree-of-freedom Prototyping Airplane Interaction Research Simulation (2PAIRS). While the 2PAIRS tool technically models more than two degrees-of-freedom of the airplane, the tool is limited to two-axis motion for any given maneuver, i.e., vertical climb, vertical descent, or level turn individually. The 2PAIRS tool was developed in the MATLAB ${ }^{\circledR}$ programming environment.

The 2PAIRS simulation tool was developed to analyze the interaction between two airplanes involved in a single encounter. The airplane performance model is based upon simplified two-dimensional airfoil theory with finite-wing approximations. These assumptions allow for a simple model that includes performance limitations while still running fast enough for a comprehensive study of the overall design space. The UAS performance in 2PAIRS is limited by either aerodynamic/propulsive limitations, maneuver command limits, or a combination of both. Note that current UAS inner-loop controllers generally limit maneuvers far below airframe capability. The variation in commanded rates used in this study models those limitations in 2PAIRS as well as cases with commands greater than the UAS can sustain.

\section{A. Descent Avoidance Maneuver Simulation}

The simulation initializes each run with two airplanes on a collision course, the UAS and a non-maneuvering intruder, as depicted in Figure 1 for a level, co-altitude intruder. In other cases herein the intruder may be flying level or given a constant rate of climb or descent. It may approach the UAS's initial trajectory through $360^{\circ}$ of azimuth, although a more limited azimuth range that accounts for right-of-way rules and symmetry was used in the 
current study. At time zero, the UAS initiates the commanded maneuver and attempts the maneuver, but may be limited by its maneuver performance envelope. The UAS will transition to the commanded descent based on load factor limit or aerodynamic limitations. It will reach and sustain the rate as long as performance allows. If it has

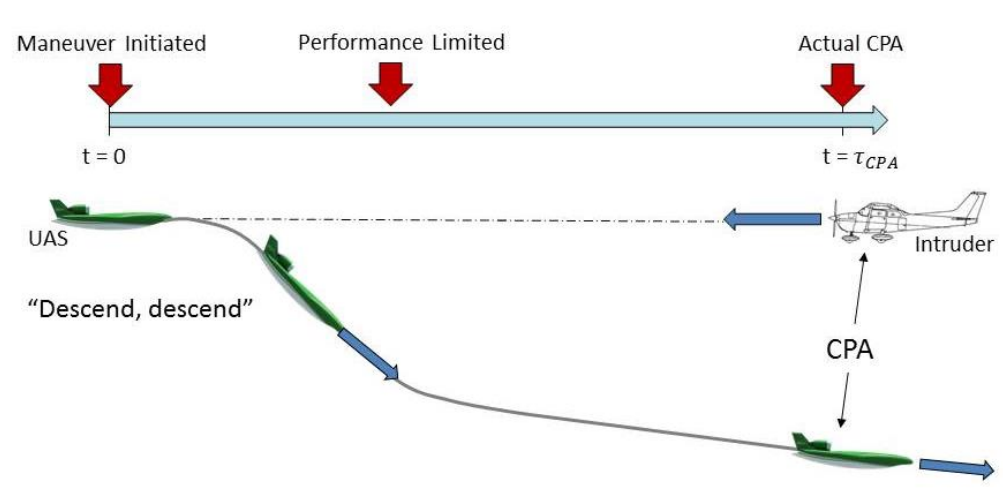

Figure 1. 2PAIRS encounter timeline. insufficient performance for the commanded maneuver, it will transition to its best sustainable performance over time based on its unique capabilities and envelope limitations. For example, an airplane that cannot sustain a commanded descent rate due to reaching its velocity not-to-exceed $\left(V_{N E}\right)$ will attempt to reach the descent rate, and if it reaches the commanded descent rate, it will sustain the rate with minimum thrust as long as possible while gaining speed. When the maximum speed, $V_{N E}$ herein, is reached, the

airplane will reduce the descent rate to sustain that speed. The simulation measures the CPA between the UAS and intruder and stops the run just after CPA is passed. The simulator's graphical user interface (GUI) version provides time histories of the UAS and intruder states, while a batch interface compiles initial states, states at CPA, and selected other mid-point states of interest. The simulation uses discrete time interval integration of the related equations of motion at $20 \mathrm{~Hz}$.

\section{B. Verification and Validation of Simulation}

Before conducting the simulation study, a verification and validation of the simulator tool was conducted. As described in Ref. 5, data representative of a set of specific individual airplanes were simulated and time histories examined to ensure that the simulated

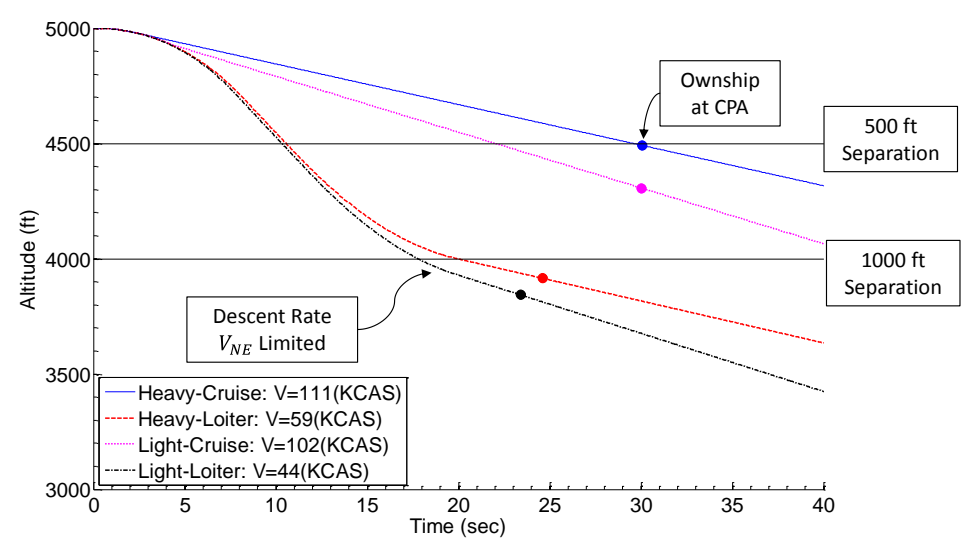

Figure 2. Descent Performance at Various Flight Conditions. Head-on, co-altitude intruder. maneuver performance in various scenarios closely matched the aerodynamic and propulsive potential to maneuver of the airplanes and modeled expected behavior of how these airplanes could react in the scenarios. It does not currently account for UAS flight control system-imposed limits other than the commanded rates varied in this study. Figure 2 shows an example of time histories for a single airplane and commanded descent rate modeled with different weights and initial speeds to examine descent maneuver behaviors. The four configurations shown in the figure represent a single airplane initially trimmed at various stages of its flight envelope. As expected, the heavy-fast configuration was the most performance limited on descent and had the lowest resulting CPA distance, followed by the light-fast configuration. The configurations with slower initial velocities were able to descend at the commanded rate longer before $V_{N E}$ was reached and achieved larger CPA values.

\section{CPA Ranges of Interest}

The community has yet to determine the CPA ranges of interest for UAS SAA. In order to scope the test matrix and to analyze and present the simulation results, a reasonable range for CPAs of interest was determined, noting that results need to include any possible CPA range that may be determined to be of interest in the future. The FAA $2^{\text {nd }}$ SAA Workshop Report (Ref. 2) describes notional boundaries (Figure 3) but only associates numbers with the 
NMAC volume. The report provides descriptions but no values for the Collision Avoidance Threshold (CAT), Well Clear Volume (WCV), or Self-Separation Threshold (SST). Note that the definitions of CAT and SST include "last chance to maneuver to avoid" the next boundary. Thus, these two boundaries are encounter and airplaneperformance dependent.

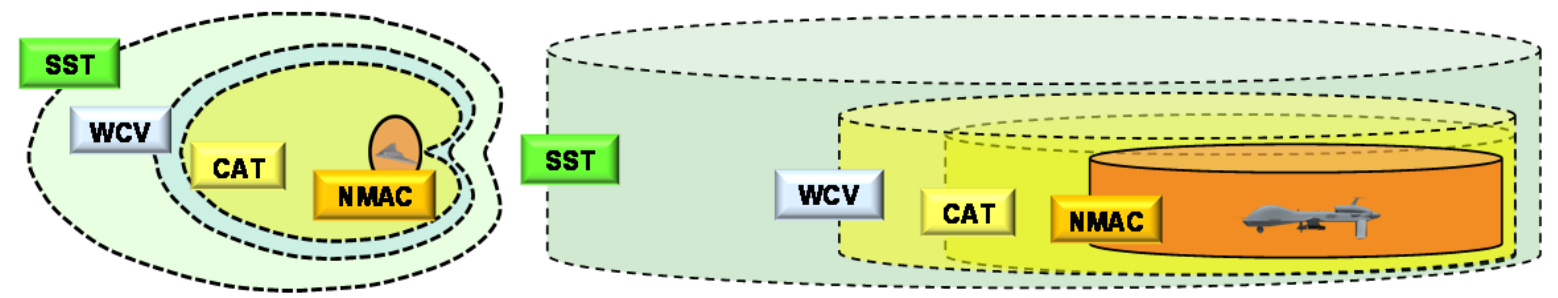

Figure 3. Figure from FAA $2^{\text {nd }}$ SAA Workshop (Ref. 2) showing collision avoidance and self-separation boundaries.

In order to draw conclusions from the results, there is a need to determine what CPA values would mean success, including both successfully avoiding a collision and maintaining well clear or self-separation. Thus, both small and large CPAs are of interest. For manned aircraft, the NMAC volume has been accepted by the aerospace community to be a cylinder defined as $\pm 100 \mathrm{ft}$ of the intruder vertically with a horizontal radius of $500 \mathrm{ft}$.

The WCV is much more difficult to define. The current regulations of CFR 14 were used to gain some insight on what an upper limit is likely to be. CFR 14 Part 91.159 and 91.179 (Ref. 1) state that aircraft operating under Instrument Flight Rules (IFR) on easterly headings are to fly at odd thousands of feet Mean Sea Level (MSL), while ones on westerly headings fly at even thousands of feet MSL. Visual Flight Rules (VFR) aircraft are required to use the same even/odd thousands of feet altitudes, but add $500 \mathrm{ft}$ as shown in Figure 4. The current airspace allows these aircraft to pass with these vertical separation distances and no horizontal separation. Thus, it seems reasonable to assume that either 500 or $1000 \mathrm{ft}$ vertical separation is the current definition of vertical well clear. As a result, this study highlights CPA results in relation to three values of vertical separation: 1$) \leq 100 \mathrm{ft}$ for NMAC; 2 ) $\leq 500 \mathrm{ft}$ referred to as IFR-VFR; and 3) $\leq 1000 \mathrm{ft}$ referred to as IFR-IFR or VFR-VFR. CPAs greater than $1000 \mathrm{ft}$ are considered to have exceeded the WCV requirements likely definition.

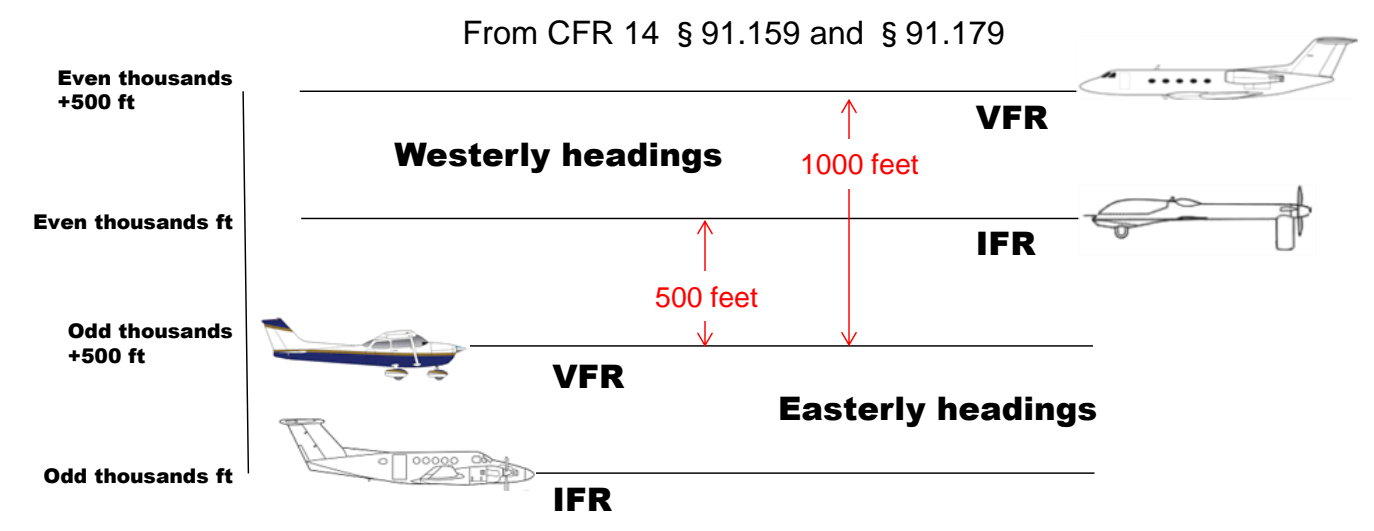

Figure 4. Directional Altitude Information Used to Select Maximum CPAs of Interest.

\section{Simulation Parameter Values}

The parameter values used in simulation of the descent maneuver are shown in Table 1. Since the goal of the simulation was to evaluate UAS performance in conducting avoidance maneuvers across a wide range of UAS, the UAS maneuver performance was modeled by varying key airplane design parameters across applicable ranges. Thus, the results can be readily applied to existing and new UAS designs. The parameters chosen are essentially for a "unit" airplane; therefore, given the same set of parameters, the performance of the resulting airplane can be estimated without knowing the size of the airplane, i.e., a wing span of $6 \mathrm{ft}$ or a wing span of $200 \mathrm{ft}$ with the same set of parameters used here would have the same performance. 
The chosen range for wing loading of $1-25 \mathrm{lbs} / \mathrm{ft}^{2}$ excludes UAS that fly very fast. Those aircraft will be the subject of later studies. A low range was chosen for the ratio of thrust to weight because UAS with higher thrust-toweight values are not the focus of this study as they would be able to outperform the airplanes of interest and therefore do not represent a limiting case. Wide ranges of aspect ratio and maximum lift-to-drag ratio values were chosen, representing nearly all existing airplanes except a limited set of veryhigh-performance sailplanes. The range chosen for maximum coefficient of lift is representative of airplanes .flying at normal cruising conditions, i.e., no blown-flaps or high-lift devices. Initial percent of maximum coefficient of

Table 1. Design Space Overview for Descent Maneuver

\begin{tabular}{|c|c|c|}
\hline Parameter & Parameter Symbol & Values \\
\hline Wing loading (Ibs/ft ${ }^{2}$ ) & $\frac{W}{S}$ & $1,9,17,25$ \\
\hline Thrust-to-weight ratio & $\frac{T}{W}$ & $0.1,0.2,0.3,0.4$ \\
\hline Aspect ratio & $A R$ & $5,16.67,28.3,40$ \\
\hline Maximum coefficient of lift & $C_{L_{\max }}$ & $0.6,1.3,2$ \\
\hline $\begin{array}{c}\text { Initial percent of } \\
\text { maximum coefficient of lift }\end{array}$ & $\% C_{L_{\max }}$ & $8,44,80$ \\
\hline Maximum lift-to-drag ratio & $\frac{L}{D_{\text {max }}}$ & $5,20,35,50$ \\
\hline Load factor Limit & $g_{\text {limit }}$ & $1.25,3$ \\
\hline Altitude (ft MSL) & $h$ & $1000,6666.7,12333,18000$ \\
\hline $\begin{array}{c}\text { Commanded descent rate } \\
\text { (ft/min) }\end{array}$ & $h_{\text {command }}$ & $500,2000,3500,5000$ \\
\hline Intruder velocity (KCAS) & $V_{\text {intr }}$ & $50,150,250$ \\
\hline Intruder vertical rate & $\dot{\mathrm{h}}_{\text {intr }}$ & $-5000,-2750,-500,0,500$, \\
\hline Intruder azimuth (deg) & $\beta$ & $0,450,5000$ \\
\hline Time-to-go (seconds) & $\tau$ & $10,20,30, \ldots 120$ \\
\hline Velocity Not to Exceed & $V_{N E}$ & $(1.15$ or 1.3$){ }^{*} V_{\text {cruise }}$ \\
\hline
\end{tabular}

lift, which was used to define the initial velocity of the airplane in each simulation run, spanned from a nominal cruise condition ( $8 \%$ of $C_{L_{\max }}$ ) to a loiter condition. Loiter is a relatively low speed and energy state that maximizes endurance and is typical of many current UAS missions. For the vertical descent avoidance maneuver, the maximum load factor, which limits the change of the flight-path angle vertically, was varied from 0 to $.75 \mathrm{~g}$. Zero g and negative $\mathrm{g}$ values are not sustainable by most airplanes primarily because of fuel and oil systems. For the vertical maneuvers, the 0.25 increment either side of $1-\mathrm{g}$ straight and level flight is what TCAS expects a pilot to use for transition from one 1-g condition to another.

The parameter ranges defining the encounter and the commanded descent maneuver were designed to cover the range of likely scenarios to be encountered by UAS while accounting for right-of-way rules. Altitude was used primarily to specify the air density for the encounter. Altitude has a direct effect on the difference between the calibrated airspeed, which drives aero-propulsive performance, and true airspeed, which dictates the inertial reference frame. The test range chosen for the encounters ranges from 1,000 to 18,000 ft MSL, which is generally landing pattern altitude to the bottom of Class A airspace. This range follows the RTCA SC-228 Terms of Reference which outlines that the focus of initial work will be on UAS transitioning to and from Class A airspace [4] or special purpose areas. The commanded descent rate for the avoidance maneuver, which the airplane receives at time $=0$ seconds in the simulation, ranged from 500 to $5000 \mathrm{ft} / \mathrm{min}$. The airplane will attempt the commanded descent rate while trying to maintain forward speed by reducing throttle. If forward speed approaches the $\mathrm{V}_{\mathrm{NE}}$, the airplane will adjust its flight path to maintain the limiting speed, as described earlier. The intruder flies at a constant velocity throughout the simulation. The speed of intruders ranged from 50 to $250 \mathrm{KCAS}$ for the full range of altitudes tested. The speed limit in the NAS below 10,000 ft MSL is 250 KCAS. While faster intruders can be encountered above that altitude, aircraft above 10,000 ft MSL are required to have transponders so they are very likely to be dealt with by air traffic control (ATC). Still, faster intruders should be assessed at a later date. Like velocity, intruder vertical rate was also held constant throughout a simulation run, ranging from a constant climb at $5000 \mathrm{ft} / \mathrm{min}$ to a constant descent at $5000 \mathrm{ft} / \mathrm{min}$. It is assumed that the intruder can maintain the specified vertical rate, and therefore, no performance limitations are considered for the intruder. While the full range of intruder climb and descent rates were run, a post-analysis exclusion was placed on the maximum flight-path angle from which the intruder could approach. For the data presented herein, the intruder did not approach at a vertical flight-path angle with magnitude greater than $\pm 12^{\circ}$. For instance, this limitation excludes $50 \mathrm{KCAS}$ intruders climbing or descending at $5000 \mathrm{ft} / \mathrm{min}$, which results in an extreme and very atypical flight-path angle of $80^{\circ}$.

6

American Institute of Aeronautics and Astronautics 
The intruder azimuth, which defines the angle of the encounter, was limited to the forward quadrants of the UAS in order to avoid scenarios with the UAS being overtaken. These scenarios were omitted because, in the current airspace, it is always the duty of the overtaking aircraft to avoid the slower-moving aircraft, and this rule is currently expected to carry over to UAS. The intruder was always heading toward the UAS as well, thus overtaking scenarios by the UAS were not included in the results to date. They would result in lower closure rate and thus require lower maneuver performance than the scenarios evaluated.

Time-to-go is the predicted time until CPA between two airplanes involved in an encounter. Since the initial $\tau$ range must encompass Collision Avoidance and Self-Separation thresholds, $\tau$ was varied from $10-120$ seconds in the simulation.

$\mathrm{V}_{\mathrm{NE}}$ is the maximum velocity the airplane can reach, which can be a structural limit, a control law limit, or a velocity restriction imposed by rules of the NAS (i.e., airplane speed limit). Given that $\mathrm{V}_{\mathrm{NE}}$ cannot be derived from aerodynamics, this study used multiples of cruise speed as $\mathrm{V}_{\mathrm{NE}}$. Two values were used: $115 \%$ and $130 \%$ of cruise speed.

\section{E. Maneuver Performance Parameters}

Analysis of this multi-dimensional design space is an iterative process with a goal of characterizing the relationship between design and performance parameters to CPA values across the range of airplanes and encounters evaluated. In order to deliver the most usable information for both UAS designers and the regulators developing certification requirements to allow UAS regular access to the NAS, performance parameters that are readily available, practical, and meaningful were sought. Maneuver performance for a given encounter is a function of the airplane's design parameters and initial condition. Thus, performance parameters that are derived from the design parameters and initial flight condition were anticipated to be more useful than design parameters alone because they relate more directly to the airplane's maneuverability and, therefore, its ability to increase CPA for a given encounter. Performance parameters found to be important to the descent maneuver are briefly described below.

\section{Specific Excess Power $\left(P_{S_{i}}\right)$}

The energy state of an airplane is described by the combination of speed and altitude: kinetic and potential energy, respectively. Specific excess power $\left(P_{S}\right)$ is a measure of the ability to change energy state [9], i.e., increase or decrease velocity, altitude, or a combination of both. Thus, the performance parameter reflects the maneuverability margin of a given airplane from a given flight condition. Specific excess power can be represented mathematically in many forms. A relatively simple one was suitable for this work:

$$
P_{S}=\frac{T-D}{W} * V=V * \sin (\gamma)=\dot{h}
$$

This equation can be expanded to directly use the design parameters from Table 1:

$$
P_{S}=\left(\frac{T}{W}-\frac{\pi e A R}{4 \frac{L^{2}}{\bar{D}_{\text {max }} C_{L}}}-\frac{C_{L}}{\pi e A R}\right) * \sqrt{\frac{2 \frac{W}{S}}{\rho C_{L}}} .
$$

If $P_{S}$ is a negative quantity, the aircraft will have to lose energy either by reducing speed (kinetic energy), decreasing altitude (potential energy), or a combination of both. During a descent, an airplane must reduce its throttle setting in order to maintain initial velocity. Otherwise, the loss of potential energy due to the reduction in altitude will result in a gain of kinetic energy in the form of speed. For an airplane trimmed to straight, level, unaccelerated flight $(T=D)$, the excess power available to change from the initial energy state is zero if the throttle is not changed. Thus, the initial $P_{S}$ is not as useful as the potential of the airplane to change energy state. For this reason, $P_{S}$ calculations for the analysis of the descent maneuver were made using minimum $\frac{T}{W}$ values, which, for this study, equal zero. Under this assumption:

$$
P_{S_{i-\min }}=\left(0-\frac{\pi e A R}{4 \frac{L^{2}}{\bar{D}_{\text {max }} C_{L}}}-\frac{C_{L}}{\pi e A R}\right) * \sqrt{\frac{2 \frac{W}{S}}{\rho C_{L}}} .
$$


Note that the value of $P_{S_{i-\min }}$ is negative for all initial conditions in this paper. The greater the magnitude of $P_{S_{i-\min }}$ the less the airplane will accelerate for a given descent rate. If the magnitude is sufficient, the aircraft may be able to descend without accelerating. For the analysis of the descent maneuver, references to $P_{S_{i}}$ refer to $P_{S_{i-\min }}$ as calculated in Eq. (3).

\section{Change in Velocity $(\Delta V)$}

Change in velocity $(\Delta V)$ is a measure of the difference between the UAS maximum speed and initial speed. This parameter effectively measures the amount of kinetic energy an airplane configuration can gain during the maneuver. The magnitude of this parameter is related to the amount of time the airplane can maintain the commanded descent rate prior to reaching $V_{N E}$ and adjusting its flight path. This parameter may be represented mathematically as:

$$
\Delta V=V_{N E}-V_{i}
$$

As the initial velocity of the UAS approaches its maximum speed, the magnitude of $\Delta V$ decreases. As the available speed margin decreases to zero, the amount of time the aircraft can maintain the commanded descent rate decreases to zero for small $P_{S_{i} \text { min }}$ values. Conversely, as the available speed margin increases, the length of time at which the aircraft can accelerate while maintaining the commanded descent rate grows; effectively enabling further separation.

\section{Initial Percent of Maximum Velocity $\left(\% V_{N E}\right)$}

This parameter is a ratio of the UAS' initial speed to maximum speed. Similar to $\Delta V, \% \mathrm{~V}_{\mathrm{NE}}$ measures the relationship between the initial speed and maximum speed but as a ratio vs. a difference. The value of $\% V_{N E}$ is inversely proportional to $\Delta V$. The parameter is calculated mathematically as:

$$
\% V_{N E}=\frac{V_{i}}{V_{N E}} .
$$

A unitless metric, it provides a direct comparison of airplanes with various speed capabilities. For instance, a high-performance airplane with an initial velocity of $175 \mathrm{KCAS}$ and a maximum velocity of $250 \mathrm{KCAS}$ has a $\% V_{N E}$ of 70. Similarly, a slow-flying airplane initially flying at $50 \mathrm{KCAS}$ with a $V_{N E}$ of $71 \mathrm{KCAS}$ would have the same $\% V_{N E}$.

\section{Analysis of Descent Maneuver}

A goal of this study was to develop response surface models capable of accurately predicting the achievable CPA between two encountering aircraft with only the UAS maneuvering. Not surprisingly, initial analysis made it clear that a global model would not be adequately accurate. Thus, the data were divided into maneuver performance groups to facilitate characterization and, hence, prediction of UAS SAA performance from airplane configuration, encounter, and commanded maneuver parameters. This section discusses the analysis approach and results.

\section{A. Performance Groups Description}

Three maneuver performance groups were developed based on characteristic performance during the commanded maneuvers. Notional descent maneuver performance representative of each of the three groups is shown in Figure 5. The Sufficient Power Differential (SPD) group contains cases where the UAS is able to maintain the commanded descent rate throughout the encounter without gaining speed. The Insufficient Power Differential (IPD) group contains cases where the UAS cannot maintain the commanded descent rate without accelerating. The Insufficient Time to Pitch (ITP) group contains cases where the UAS is continually in the process of turning (pitching down) to the commanded descent rate throughout the simulation.

Figure 6 highlights the differences between these performance groups, showing CPA vs. $P_{S_{i}}$ for each of the groups with commanded descent rates delineated by color. It is important to recall that the performance groups are functions of the airplane configuration, encounter parameters, and the commanded maneuver. Therefore, a single airplane configuration can occupy any of the described performance groups. Equations were developed to enable the 
2PAIRS simulation tool to predict which performance group the UAS, encounter, and commanded maneuver rate are in before making each run.

Descent maneuver performance for each group is considered individually in the following subsections.

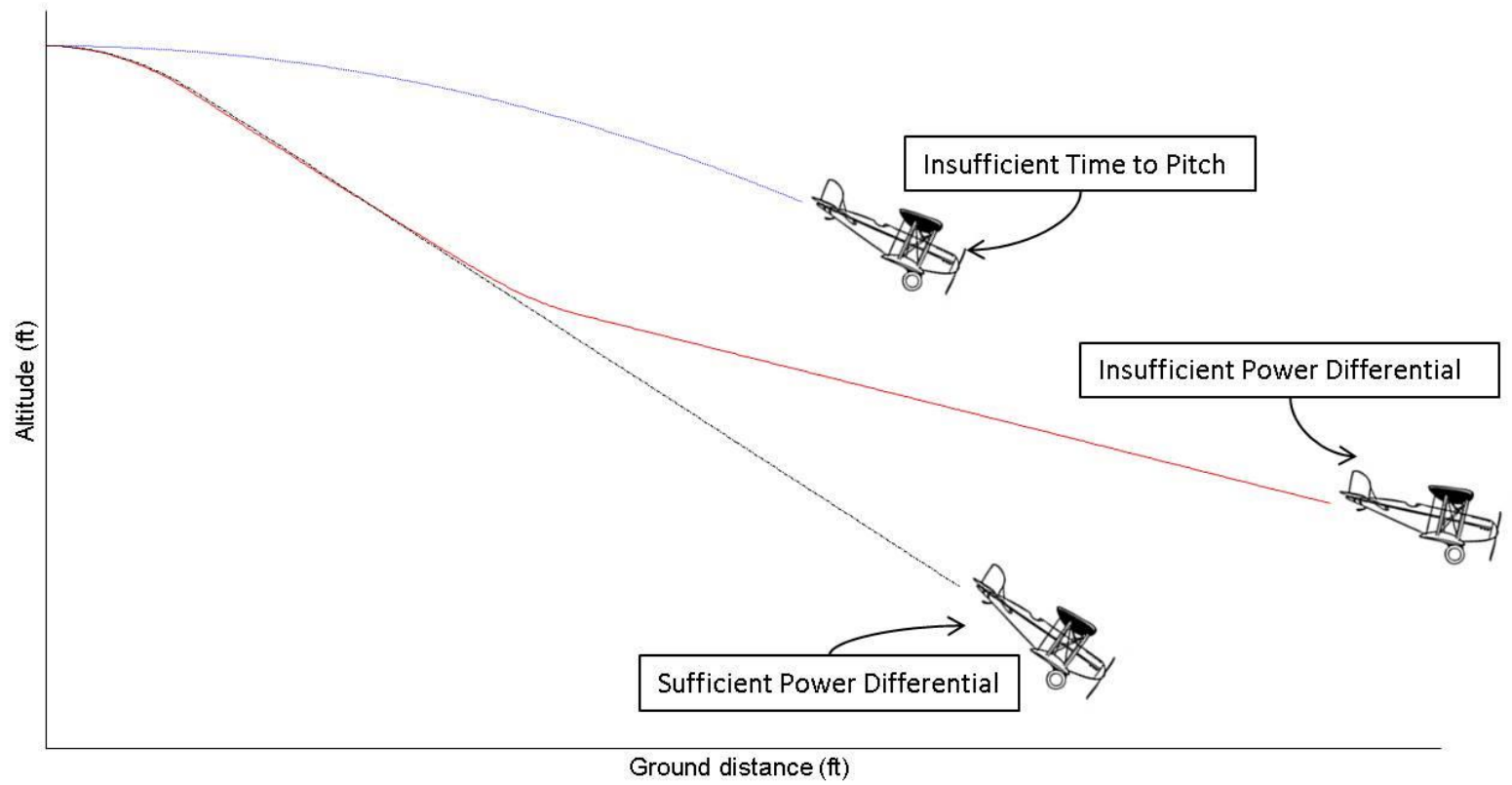

Figure 5. Notional chart depicting three descent maneuver performance groups.

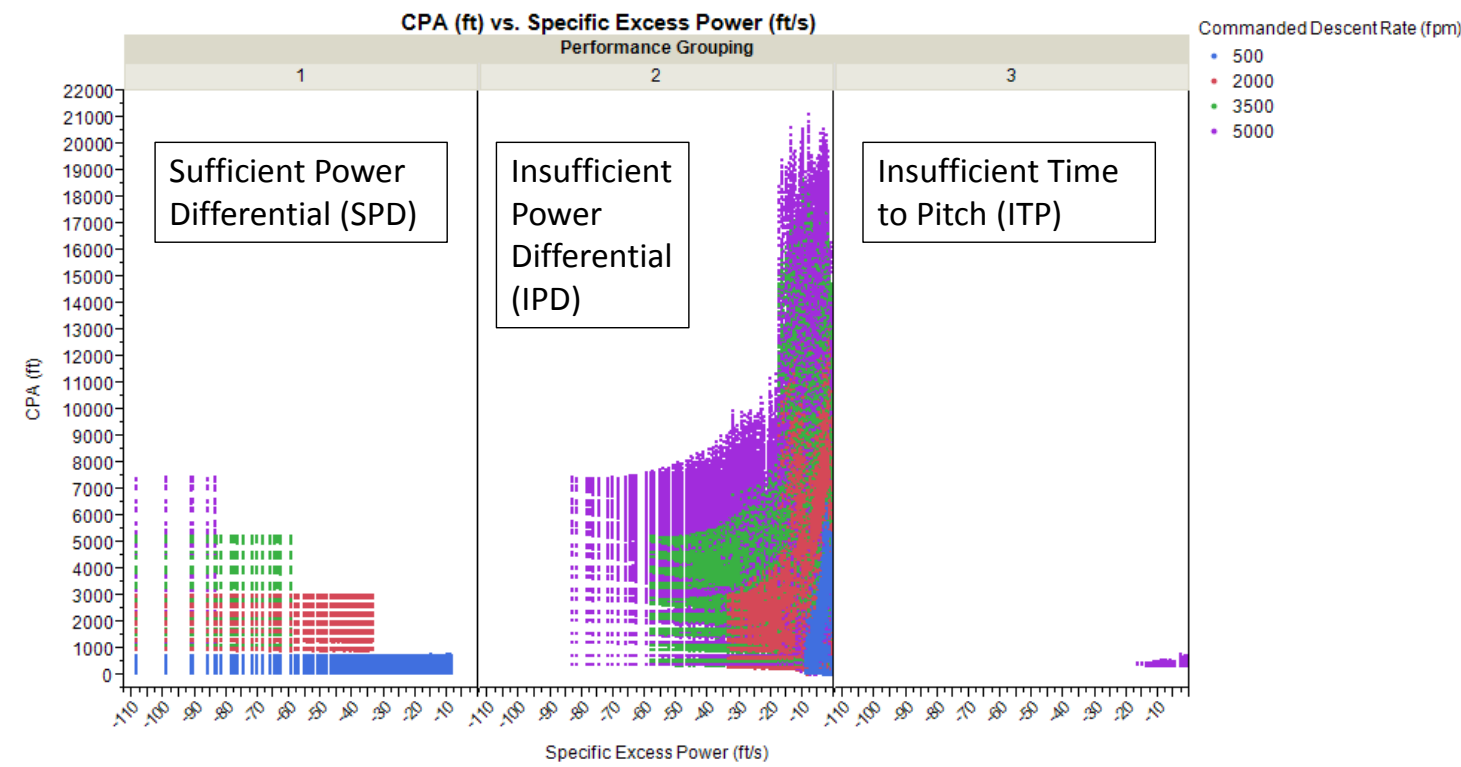

Figure 6. Grouping of Characteristic Descent Performance.

\section{B. Insufficient Time to Pitch Group Analysis}

The ITP group is characterized by airplanes that are unable to reach the commanded descent rate due to very short simulation times and/or very high speeds. This group can be modeled using simple geometry and known performance limitations (i.e., limited pitch rate and $\tau$ ). Note from Figure 6, that this performance group contains a very small number of simulation runs. Specifically, it only includes encounters with $\tau=10$ seconds and a commanded descent rate of $5000 \mathrm{fpm}$. Due to the limited variability in applicable simulation runs, simple geometric 
nature of the results, and small number of cases where this occurred, this group was not analyzed in more detail for this paper.

\section{Sufficient Power Differential Group Analysis}

Runs in which airplanes are able to sustain the commanded descent rate throughout the encounter without gaining forward speed define this performance group. As shown by the straight right vertical edge of each commanded-rate group in Figure 6, for each commanded descent rate there exists a minimum $P_{S_{i}}$ magnitude that makes constant speed possible. For instance, airplanes within this performance group with a commanded descent rate of $5000 \mathrm{fpm}$, or $83.33 \mathrm{fps}$, have a $P_{S_{i}}$ magnitude of $83.33 \mathrm{fps}$ or greater. Thus, Figure 6 shows a series of horizontal lines corresponding to $\tau$ and commanded rate for all configurations with $P_{S_{i}}$ magnitudes greater than the maximum respective value for each commanded descent rate. The relationship between $P_{S_{i}}$ and $\dot{h}_{\text {command }}$ also shows that $\dot{h}_{\text {sustainable }}$ can be calculated for any initial flight condition.

Considering the geometry of a constant-rate pitch (defined by speed and $g_{\text {limit }}$ or achievable g) and constant descent rate, the achievable CPA for airplanes within the SPD group is predictable using only three variables: $\dot{h}_{\text {command }}, g_{\text {limit }}$, and $\tau$. The parametric equation for CPA below was derived in terms of these variables from the available data:

$$
\begin{aligned}
& C P A=-843.81+17.623 * \tau-38.626 * g_{\text {limit }}+0.787 * \dot{h}_{\text {command }}+\left(\dot{h}_{\text {command }}-1058.309\right) *(0.01667 * \\
& \left.\tau-0.0727 * g_{\text {limit }}-0.5608\right) .
\end{aligned}
$$

Applying the parametric equation to a matrix of the applicable variables produces Figure 7 and Figure 8. These figures or the parametric equation can be used to determine the achievable CPA for any encounter scenario that falls within this performance group.

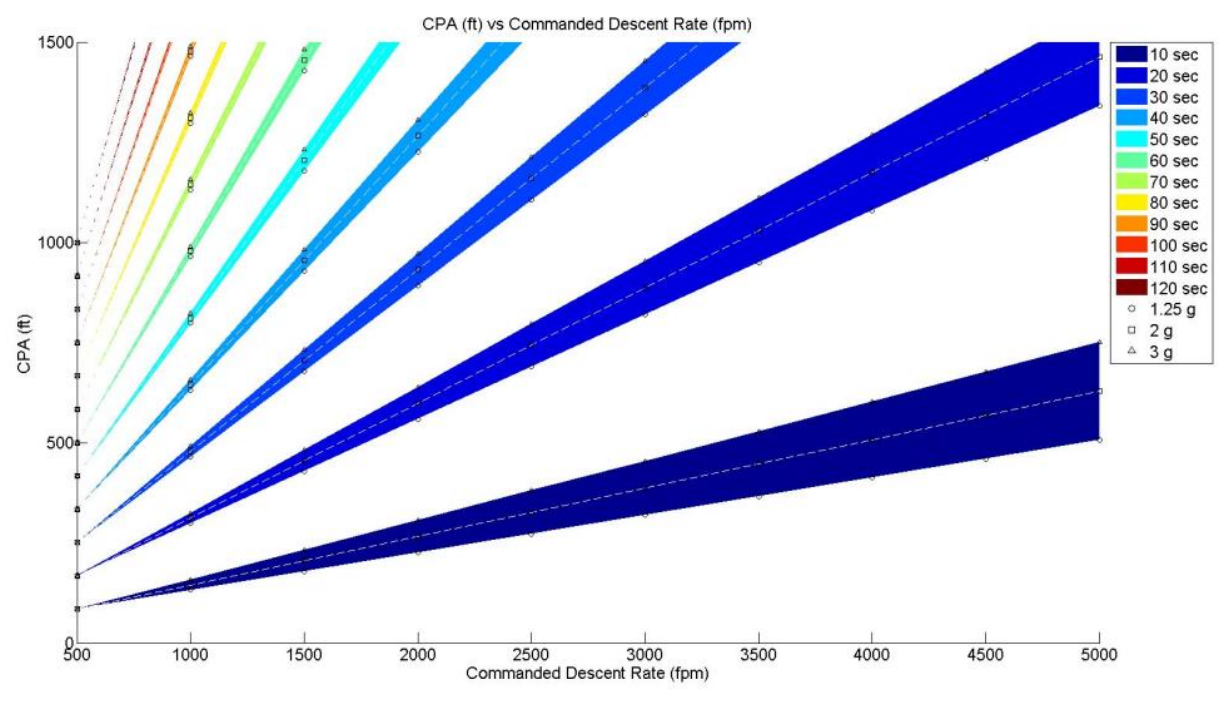

Figure 7. Application of Descent Maneuver Parametric Equation for SPD Group; CPA vs Commanded Descent Rate; $\tau$ Shown by Color. 


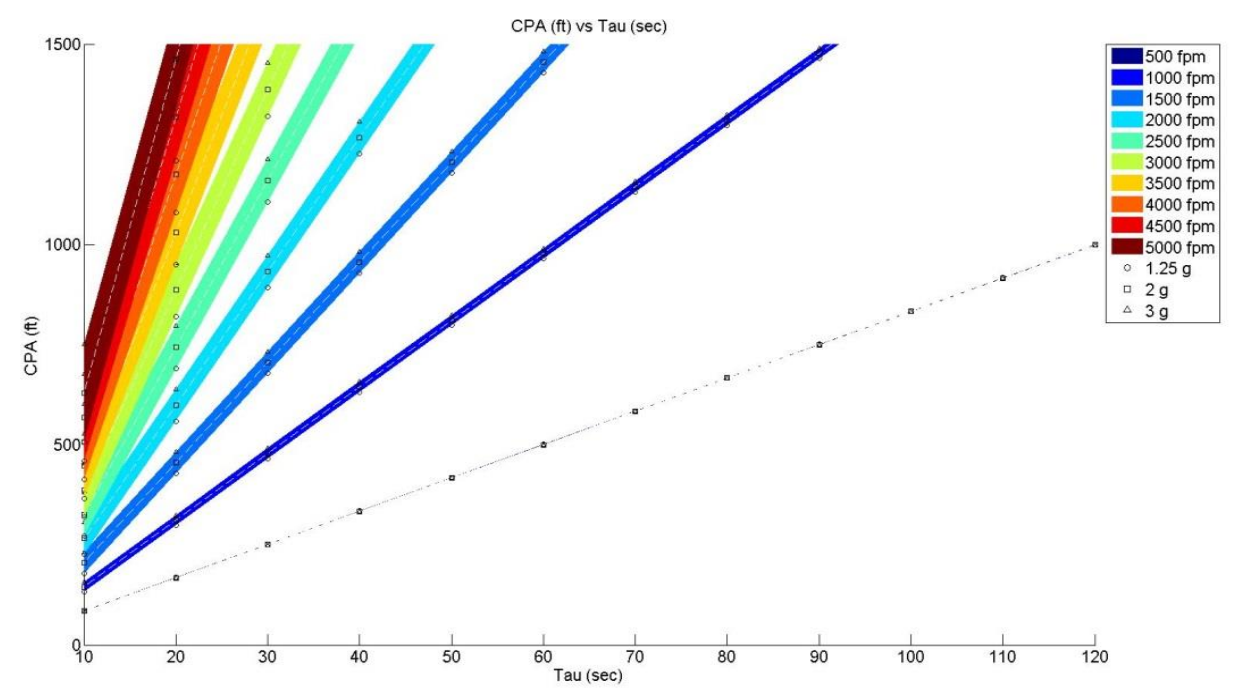

\section{Figure 8. Application of Descent Maneuver Parametric Equation for SPD group; CPA vs $\tau$; Commanded Descent Rate Shown by Color.}

\section{Insufficient Power Differential Group Analysis}

The IPD performance group is occupied by runs with airplanes unable to avoid gaining speed due to the combination of airplane performance and magnitude of the commanded descent rate. A characteristic of this group is the reduction in time to CPA due to the increase in closure rate as the UAS accelerates. The achievable separation for airplanes within this group is difficult to predict due to the variability in the amount of time the commanded rate is maintained, speed change impacting actual versus predicted $\tau$, and the sustainable descent rate associated with $V_{N E}$. The IPD group contains more results with higher commanded descent rates than the SPD group because as commanded descent rate increases fewer aircraft are able to maintain their initial speed.

There are three distinct acceleration performance characteristics within the IPD group: 1) the airplane accelerates throughout the simulation time; 2) the airplane accelerates to the configuration's $V_{N E}$ and must reduce its descent rate; and 3) the airplane accelerates to the terminal velocity associated with the configuration and the commanded descent rate. In the third case, the airplane stops accelerating during the maneuver due to physics without adjusting descent rate as the airplane's drag becomes equal to the component of gravity working against it.

An analysis of individual parameter effects on achievable CPA values for the IPD encounters follows.

\section{Effect of $\tau$ on $C P A$}

This section highlights the effect of $\tau$ on CPA. The initial positions of the UAS and intruder are defined by their respective speed, $\tau$, azimuth, and intruder elevation angle. This allows direct comparison of high-speed and lowspeed encounters because, although the range may vary greatly, the time to collision is the same. Figure 9 shows the IPD group separated by the $\tau$ value used for the simulation runs. One trivial observation to be made is that the maximum achievable separation increases with $\tau$. The point protruding left for each $\tau$ correspond to specific commanded descent rates in the data. This will be examined further in the next section. 


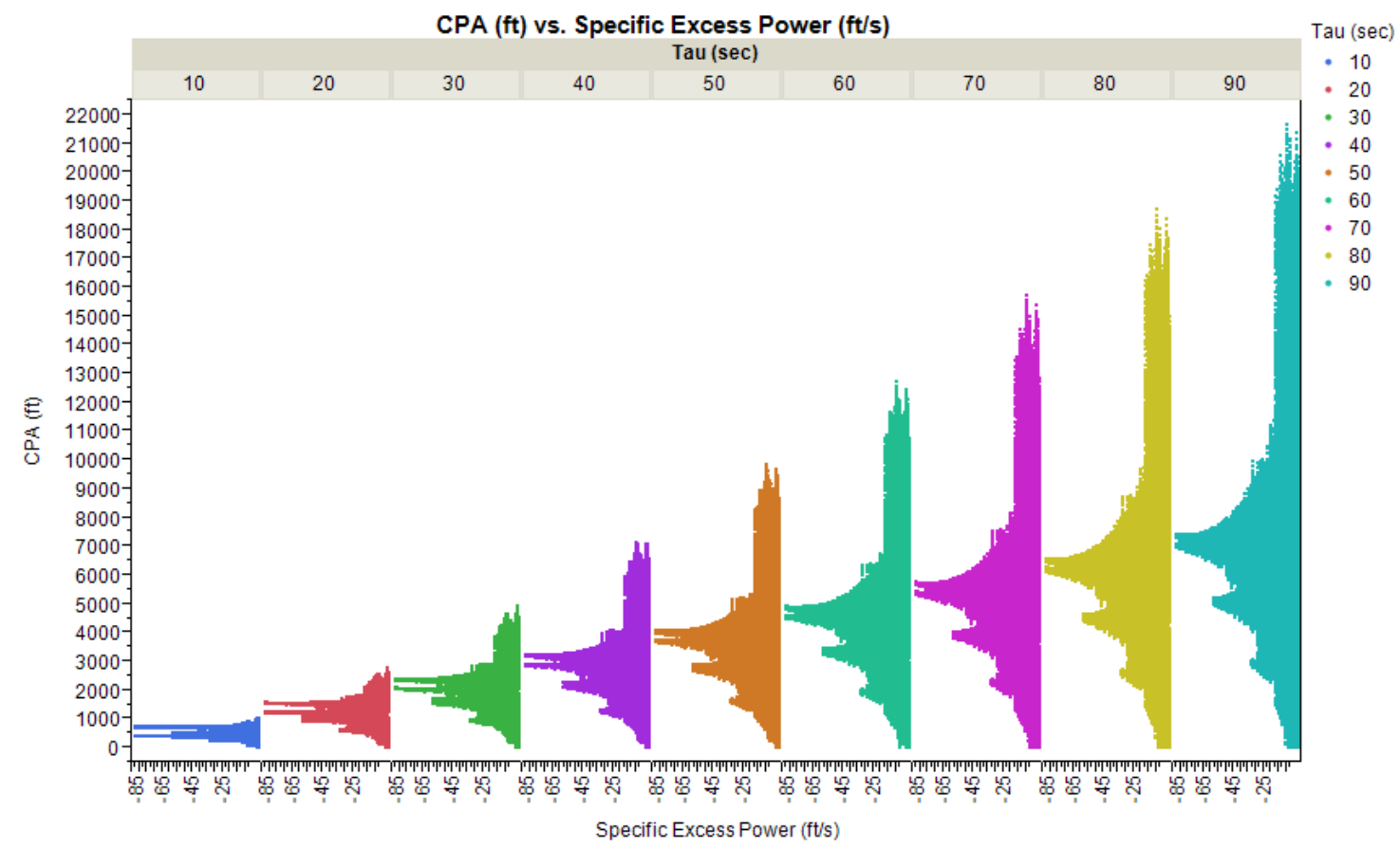

Figure 9. Effect of $\tau$ on CPA for IPD Group.

The CPA vs. $P_{S_{i}}$ characteristics for each $\tau$ value result in very similar shapes but different scales across $\tau$ values. Though any $\tau$ value may be chosen to perform the following analysis of the other parameter effects, the analysis that follows focuses on $\tau=60$ seconds.

\section{Effect of $\dot{h}_{\text {command }}$ on $C P A$}

This section breaks out the impact of $\dot{h}_{\text {command }}$ on CPA. The magnitude of $\dot{h}_{\text {command }}$ impacts how quickly the aircraft gains speed for each $P_{S_{i}}$. Figure 10 shows the Insufficient Power Differential Group with $\tau=60$ seconds and color delineating $\dot{h}_{\text {command }}$ values. From Figure 10 , there is a maximum $P_{S_{i}}$ magnitude for each $\dot{h}_{\text {command }}$ value. The maximum magnitude is the demarcation between this IPD and the SPD group. The variability in CPA collapses to the predictable value of the SPD group as the magnitude of $P_{S_{i}}$ increases. With continuously varying $\dot{h}_{\text {command }}$

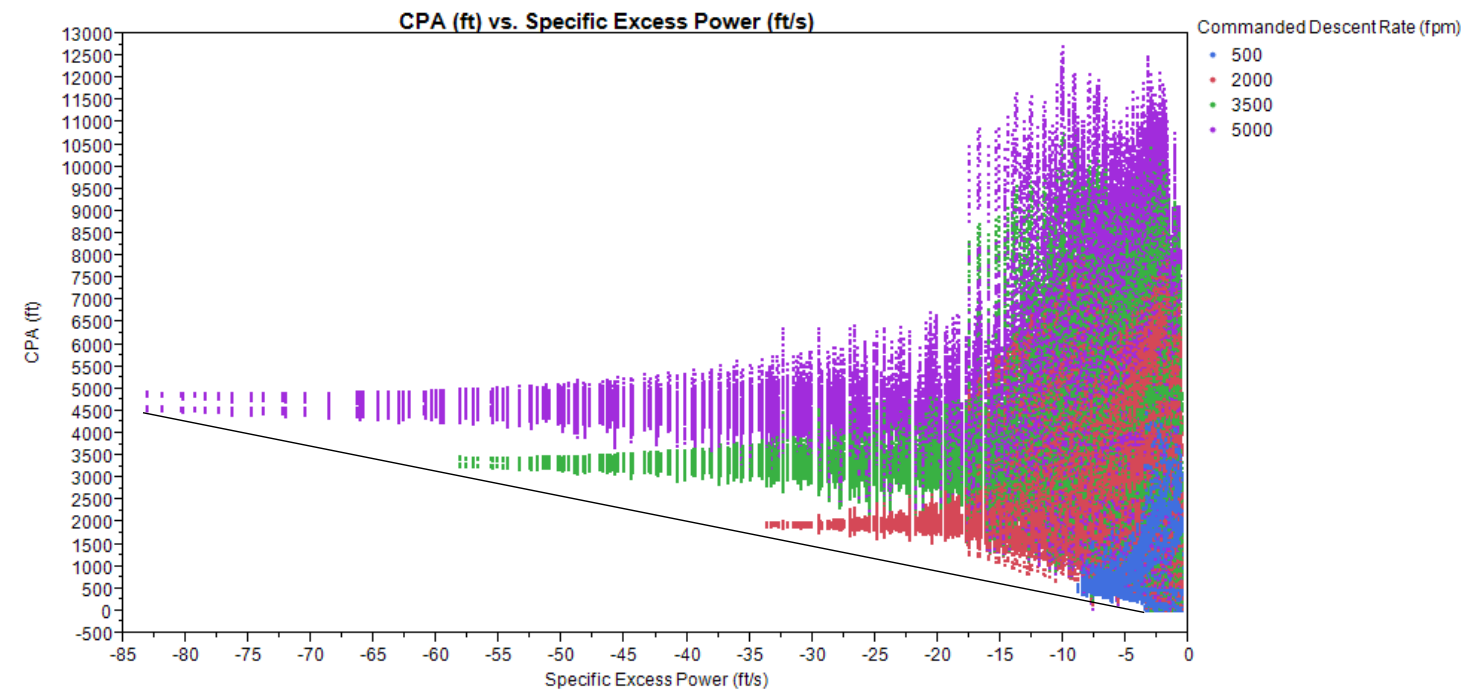

Figure 10. Effect of $\dot{\boldsymbol{h}}_{\text {command }}$ on CPA for IPD Group; $\boldsymbol{\tau}$ of 60 seconds.

12

American Institute of Aeronautics and Astronautics 
values, the maximum $P_{S_{i}}$ magnitudes would be continuous and the edge of the data would be similar to the line drawn in Figure 10.

From Figure 10, the performance of all $\dot{h}_{\text {command }}$ values are comparable but on various scales.

\section{Effect of $g_{\text {limit }}$ on $C P A$}

From Figure 10, there is a fork evident in the CPA responses of the $5000 \mathrm{fpm}$ descent configurations as $P_{S_{i}}$ magnitude increases. This split is due to the two levels of $g_{\text {limit }}$ used in the data matrix and occurs for the other commanded rates, though it is not evident at the scale of the figure. For a given $P_{S_{i}}$, the greater CPA value is associated with the $g_{\text {limit }}$ of 0 , while the lesser CPA value is associated with the $g_{\text {limit }}$ of 0.75 . The CPA is proportional to the $g_{\text {limit }}$ because the $g_{\text {limit }}$ determines the amount of time required to pitch to the commanded descent rate. The faster the airplane can reach the commanded rate, that is the higher the $g_{\text {limit }}$, the more time spent at the specified commanded rate resulting in more separation. The variation in CPA due to the various $g_{\text {limit }}$ values is most apparent in the configurations with $5000 \mathrm{fpm}$ descent rate. This is because the required amount of time spent transitioning to the commanded rate increases with the commanded rate.

\section{Effect of $\beta$ on $C P A$}

The intruder azimuth $(\beta)$ also affects the achievable CPA for a given encounter. Figure 11 shows the Insufficient Power Differential Group with $\tau=60$ seconds and $\dot{h}_{\text {command }}=-2000 \mathrm{fpm}$. The data are separated by $\beta$, and the color represents $\% V_{N E}$. The variation in CPA for airplanes with low $\% \mathrm{~V}_{\mathrm{NE}}$ in encounters with an intruder approaching from $90^{\circ}$ is larger than the results from the other two $\beta$ values.

As previously described, there are multiple characteristic performances within the IPD group. These characteristics are apparent in Figure 10 as the vertical lines of low $\% V_{N E}$ (blue) have different trends than the high $\% V_{N E}$ (red) values as $P_{S_{i}}$ decreases. These vertical lines of low $\% V_{N E}$ are features of aircraft that reach terminal velocity.

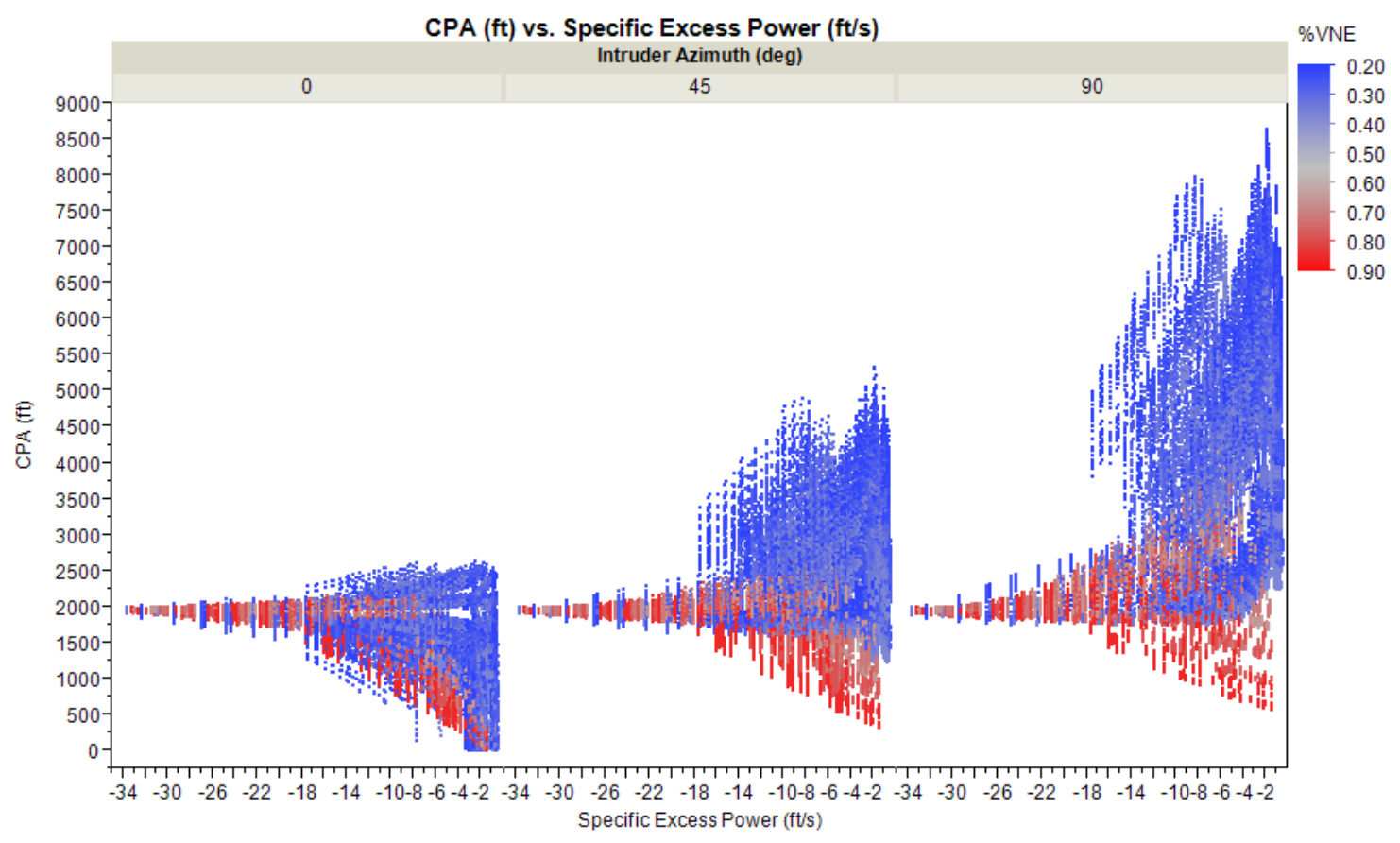

Figure 11. Effect of $\beta$ on CPA for IPD Group; $\tau=60$ seconds; $\dot{h}_{\text {command }}=-2000 \mathrm{fpm}$.

\section{Effect of $\dot{h}_{\text {intruder }}$ on $C P A$}

Figure 12 shows effect of $\dot{h}_{\text {intruder }}$ on the achievable CPA response of the IPD group as a function of $P_{S_{i}}$ separated by the intruder vertical rate for head-on cases only. Color delineates the characteristic acceleration performance described in Section D. Figure 13 shows $\% V_{N E}$ as color. The data shown is for head-on encounters 13

American Institute of Aeronautics and Astronautics 
with $\tau=60$ seconds and $\dot{h}_{\text {command }}=-2000 \mathrm{fpm}$. For the previously described SPD group, this encounter would result in a separation of $\sim 2000$ feet. From Figure 12, it is possible to achieve a separation greater than 2000 feet, if the encounter involves an intruder descending toward the UAS' flight path at a higher descent rate than the UAS' commanded descent rate with the UAS accelerating but not reaching $V_{N E}$. There are airplane configurations that reach $V_{N E}$ that are able to achieve a higher CPA. A detailed analysis revealed that these configurations accelerate, and therefore, are flying at the commanded descent rate, for the majority of the encounter time. For encounters involving a descending intruder, after the commanded maneuver is initiated, the UAS is moving away from the

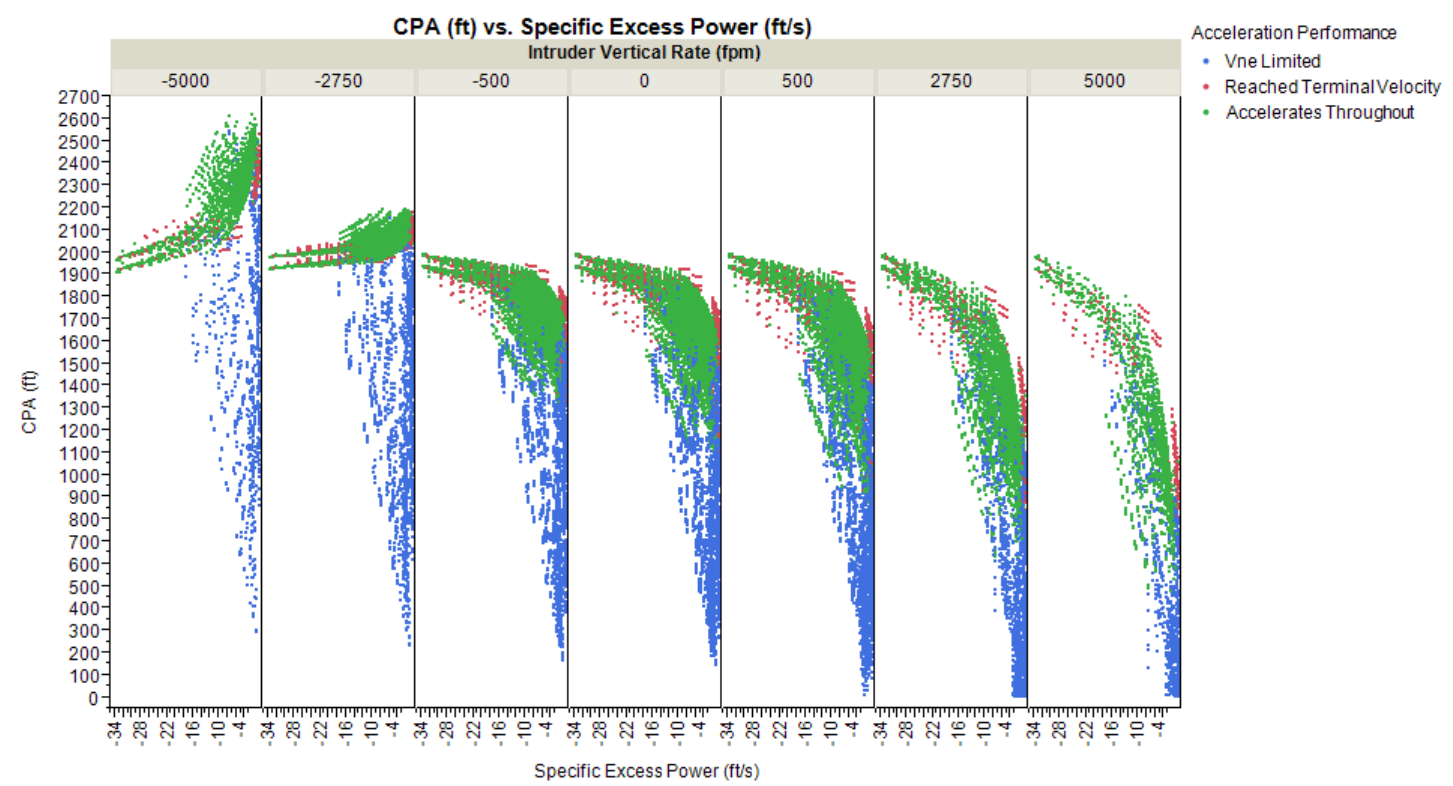

Figure 12. Effect of $\dot{h}_{\text {intruder }}$ on CPA for IPD Group; $\tau=60$ seconds; $\dot{h}_{\text {command }}=-2000 \mathrm{fpm}$; Head-on Encounter.

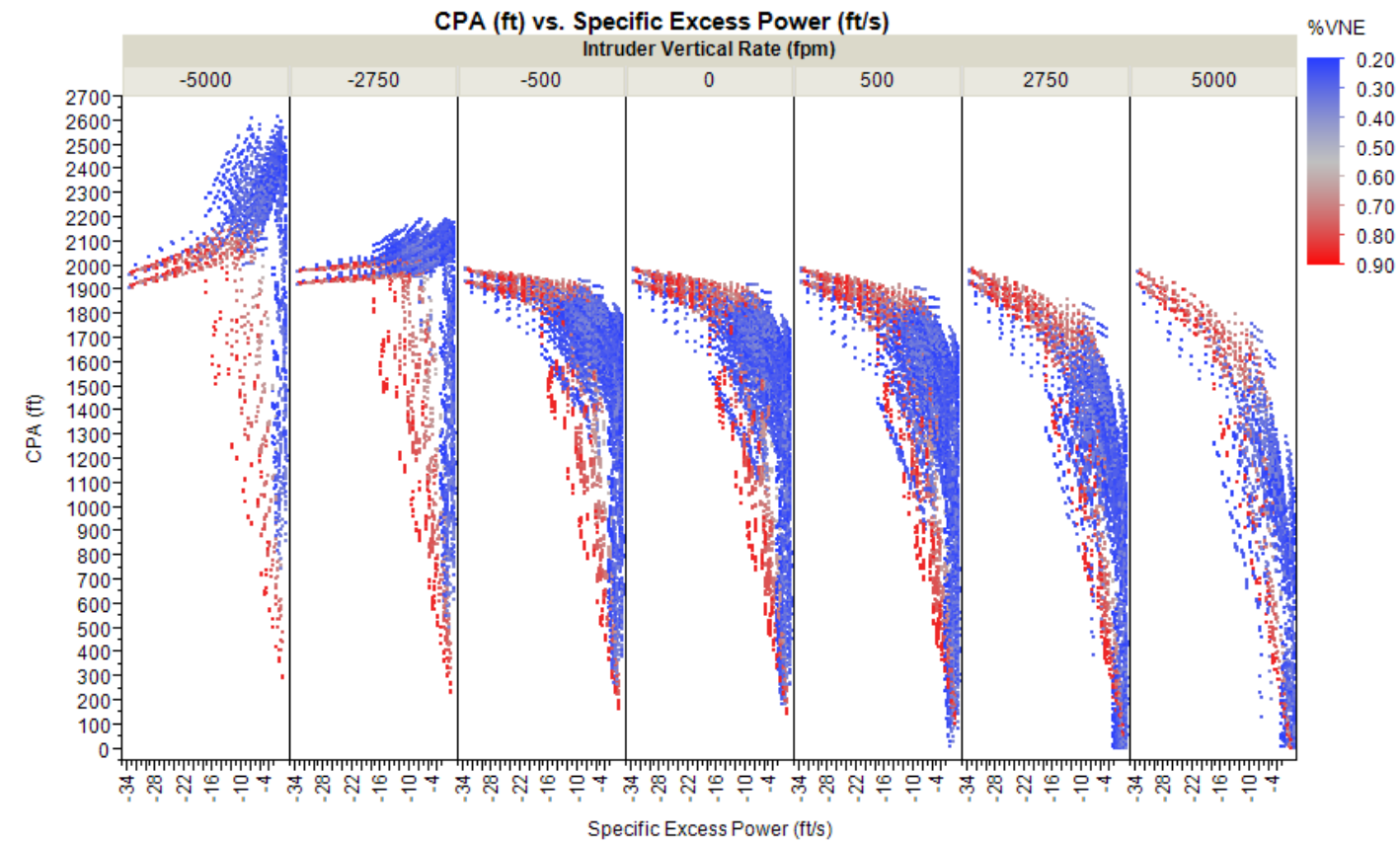

Figure 13. Effect of $\dot{h}_{\text {intruder }}$ on CPA for IPD Group; $\tau=60$ seconds; $\dot{h}_{\text {command }}=-2000$ fpm; Head-on Encounter.

14

American Institute of Aeronautics and Astronautics 
intruding aircraft. Conversely, for encounters involving intruder aircraft that are climbing, the UAS' commanded maneuver results in the UAS moving towards the intruder and thus smaller achievable CPAs.

From Figure 13, the majority of head-on encounters show minimum CPA for airplanes with a high $\% V_{N E}$. However, for encounters involving fast-ascending intruders, there exist UAS with low $\% V_{N E}$ that result in very small CPAs. These low CPA values are a result of the UAS moving towards the intruder aircraft, creating a collision after the maneuver is performed.

Figure 14 illustrates a collision after the avoidance maneuver is initiated. This figure shows a UAS initially flying at a loiter, or low $\% V_{N E}$, condition. The airplane is involved in an encounter with a climbing intruder. The UAS begins to descend and accelerates at idle power, but is limited by $V_{N E}$ and is forced to adjust to a sustainable descent rate. Due to the acceleration of the UAS, the intruder aircraft has not reached the position of the predicted collision when the actual CPA occurs. Thus, the actual $\tau$ is less than the predicted value. Due to the specific combinations of intruder trajectory and UAS descent performance, the two aircraft collide prior to the predicted collision as a result of trying to avoid it. If the UAS' collision avoidance logic is unable to anticipate the descent performance of the UAS, it may command a descent rate that results in a collision with the intruder it is trying to avoid prior to the predicted time of CPA.

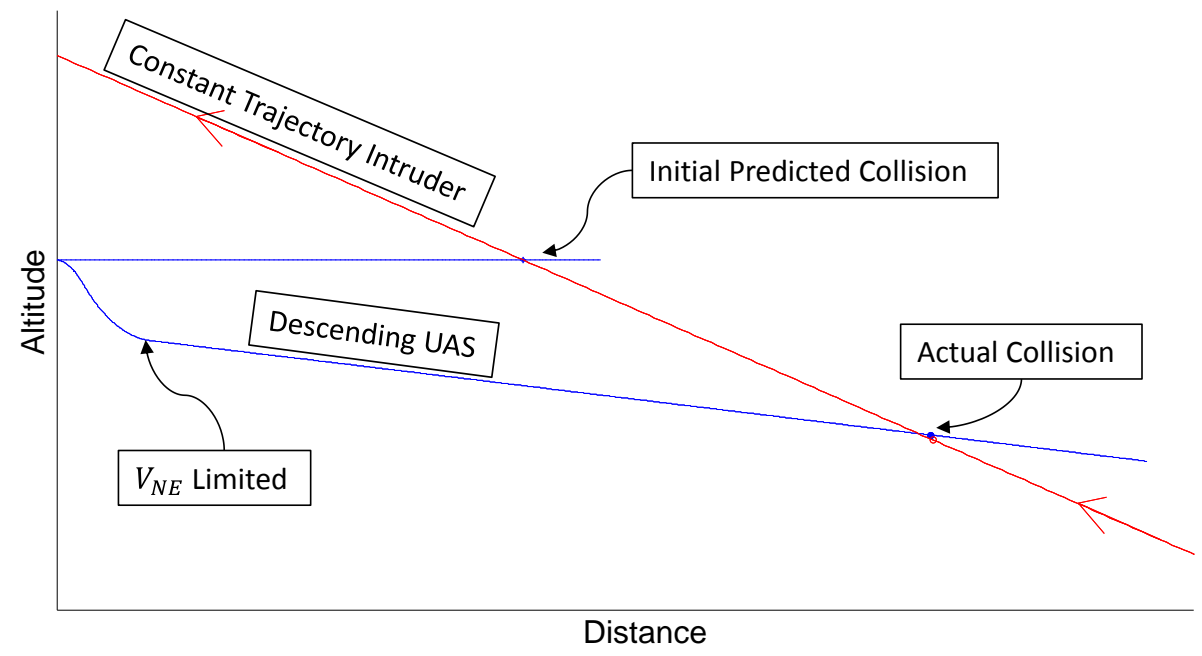

\section{Figure 14. Example of Collision Resulting from Maneuver Involving Constant Trajectory Intruder and Descending UAS.}

\section{Analysis to Predict SAA Performance Requirement for IPD Group}

The goal of this analysis is to be able to predict the UAS maneuver element of SAA performance required for a given UAS configuration and encounter scenario to achieve a desired CPA. After identifying the parameters that have a large effect on the achievable CPA, a series of performance parameter thresholds can be derived for a given encounter scenario. For instance, consider a UAS involved in an encounter with a co-altitude $\left(\dot{h}_{\text {intruder }}=0\right)$, head-on intruder $\left(\beta=0^{\circ}\right)$. The aircraft has a push-over $g_{\text {limit }}$ of 0.75 and can command as much as a $2000 \mathrm{fpm}$ descent. At its initial trimmed condition, the UAS is able to detect the intruder aircraft early enough for a maneuver to be commanded 60 seconds from collision. Assuming the UAS immediately begins a descending avoidance maneuver and is able to sustain a $2000 \mathrm{fpm}$ descent without accelerating, the UAS will achieve a separation of about 2000 feet. If, however, the UAS is unable to sustain the commanded descent rate without accelerating, it will not achieve this same separation. Now assume that a well-clear threshold has been defined as 1000 feet of separation between the two aircraft. Figure 15 shows CPA as a function of $P_{S_{i}}$. The color represents $\Delta V$ (delta_Vne in the legend). In order to guarantee this safety threshold is maintained, Figure 15 shows that the UAS must have a $P_{S_{i}}$ of $-11 \mathrm{ft} / \mathrm{s}$ or better. If the UAS is able to command a $5000 \mathrm{fpm}$ descent, Figure 16 shows that a $P_{S_{i}}$ of $-10.5 \mathrm{ft} / \mathrm{s}$ is required to meet the same threshold. 


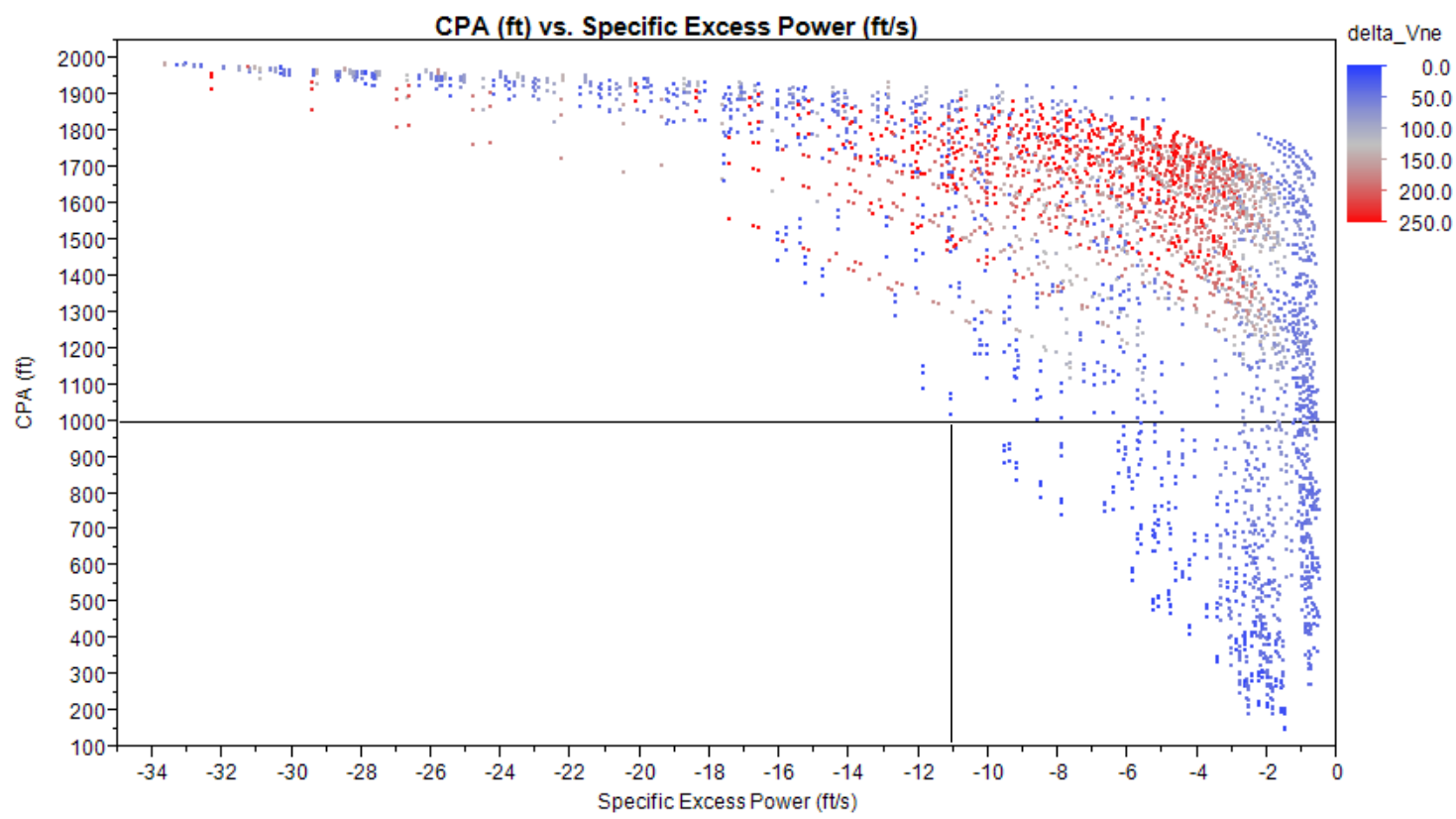

Figure 15. $\quad P_{S_{i}}$ Required for 1000-ft Separation for IPD Group with $\dot{h}_{\text {command }}=-\mathbf{2 0 0 0} \mathrm{fpm} ; \tau=$ 60 seconds; Head-on, Co-Altitude Intruder.

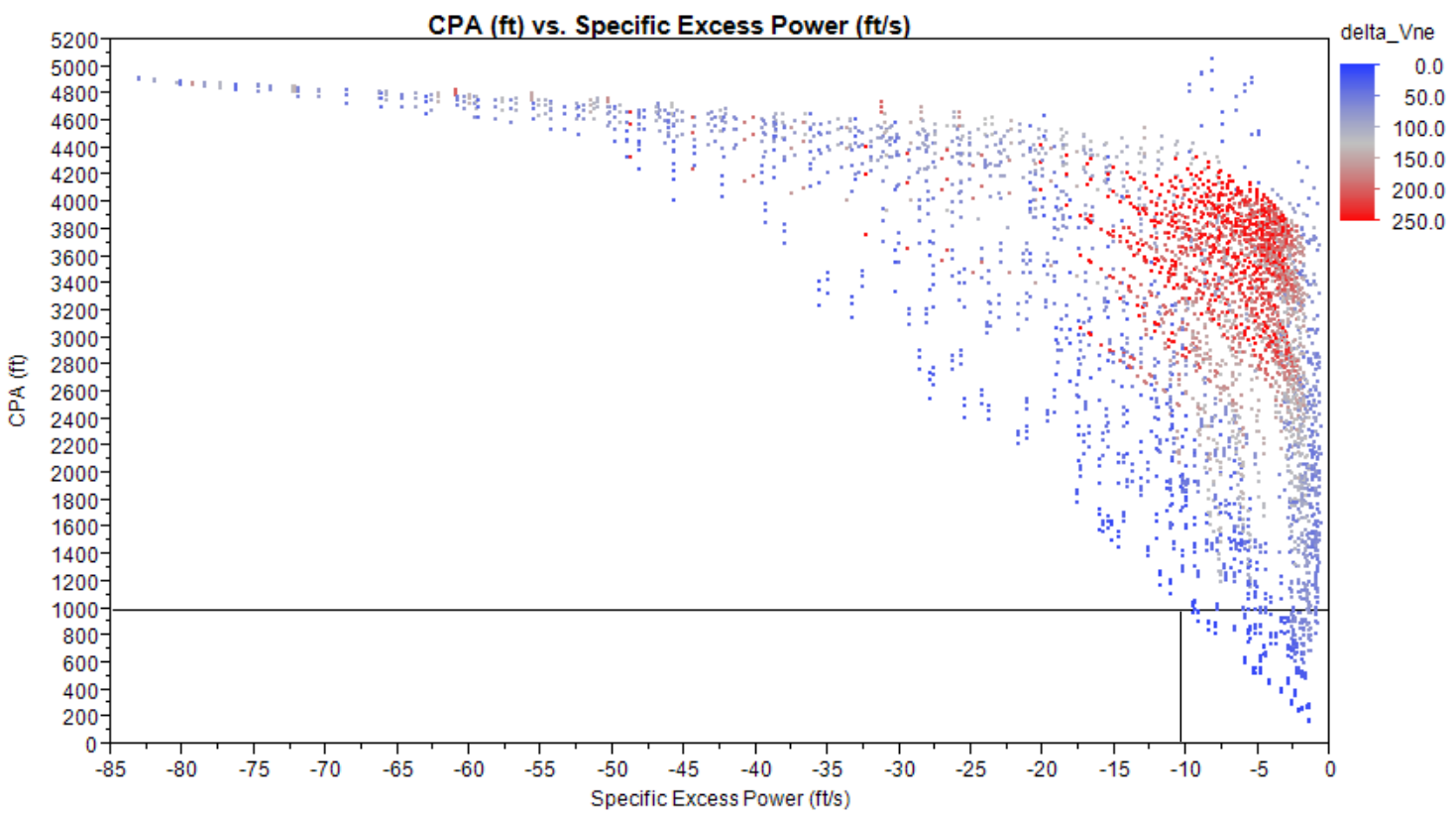

Figure 16. $\quad P_{S_{i}}$ Required for 1000-ft Separation for IPD Group with $\dot{h}_{\text {command }}=-\mathbf{5 0 0 0} \mathrm{fpm} ; \tau=$ 60 seconds; Head-on, Co-Altitude Intruder.

While these $P_{S_{i}}$ values and corresponding rates guarantee the UAS meets the specified separation threshold, it is possible to reach the same threshold with a lower $P_{S_{i}}$ magnitude as seen in both figures. Figure 17 shows simulation data with airplane configurations that have $P_{S_{i}}$ greater than $-11 \mathrm{ft} / \mathrm{s}$. The figure shows CPA as a function of $\Delta V . P_{S_{i}}$ is

16

American Institute of Aeronautics and Astronautics 
shown in the figure in color. From the figure, if an aircraft has no excess power $\left(P_{S_{i}}=0\right)$, it may achieve CPA $\geq$ 1000 feet if it is able to gain $90 \mathrm{KCAS}$ before reaching $V_{N E}$. If the aircraft has a higher $P_{S_{i}}$, an even smaller $\Delta V$ is required for the same separation to be achieved. The figure indicates that some combinations of $P_{S_{i}}$ and $\Delta V$ with smaller values than the individual requirements can meet the same CPA minimum. However, a consistent mathematical relationship had not been determined for publication herein.

This type of analysis is applicable to all encounter scenarios but depends heavily on a separation threshold being specified. Future work will include this type of analysis applied to the specific separation threshold as it is determined by industry and regulators.

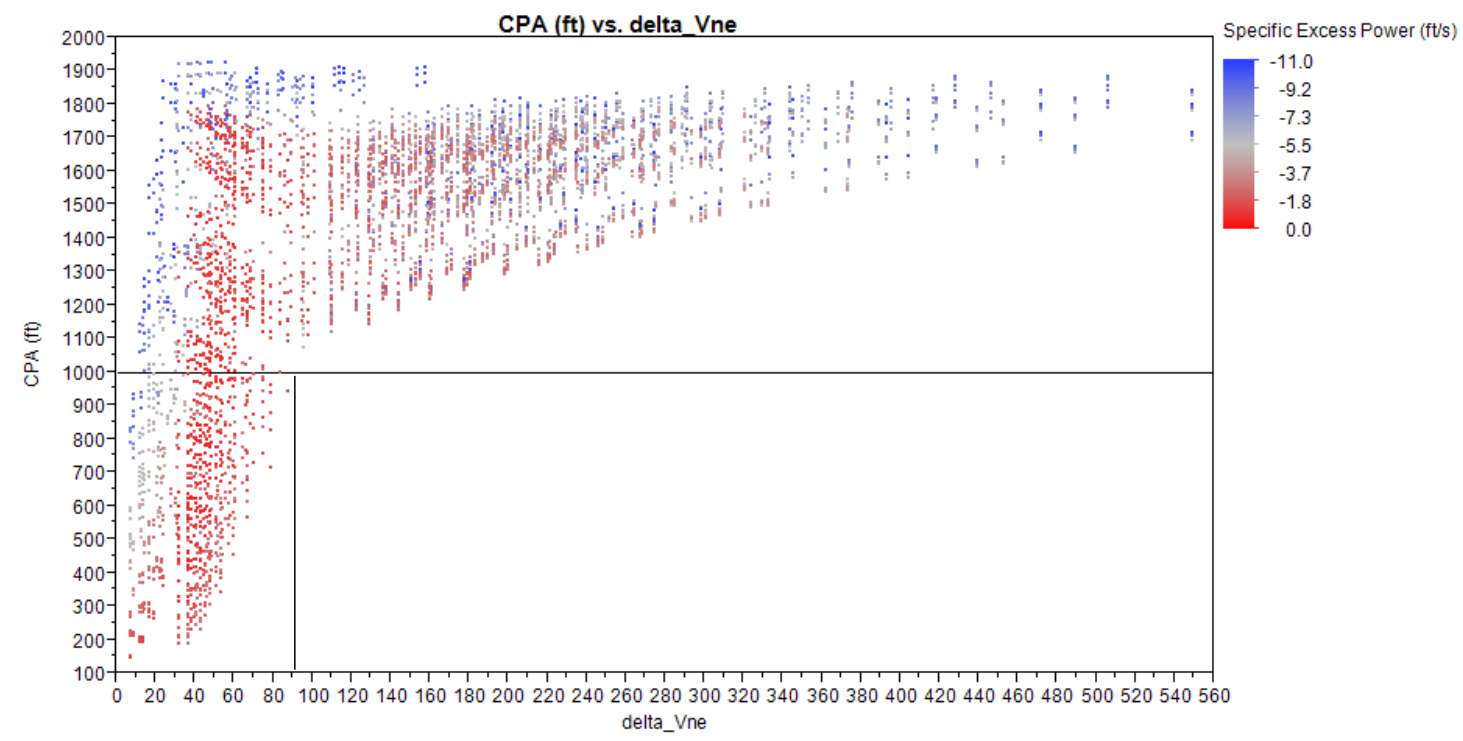

Figure 17. $\Delta V$ Required for 1000-ft Separation for IPD Group with $\dot{h}_{\text {command }}=2000 \mathrm{fpm} ; \tau=60$ seconds; $P_{S_{i}} \geq-11 \mathrm{ft} / \mathrm{s} ;$ Head-on, Co-Altitude Intruder.

\section{Conclusion}

A simulation tool was developed and a study conducted to assess the trade space of UAS descent maneuver performance capability across a broad range of encounters. The simulation tool supports evaluations of macro results from large numbers of UAS performance models and encounter geometries as well as more detailed timehistory analysis of model/encounter combinations. A verification and validation of the simulation was performed using approximate aerodynamic/propulsive performance of a series of existing airplanes. Test matrix parameter ranges for UAS aerodynamic and propulsive parameters, encounter model parameters, and intruder states were established based on existing airplanes and consistent with RTCA SC-228's near-term NAS integration focus. This study of the trade space between UAS maneuver performance and SAA system performance provides informative results, analysis, and methodologies to help regulators and UAS designers develop regulations and capabilities for safe and efficient integration of UAS into the NAS.

A detailed discussion of the impact of UAS performance on achievable CPA was presented for the vertical descent maneuver. Results demonstrated that specific excess power, $P_{S_{i}}$, of a given aircraft is a key parameter in predicting achievable CPA for the vertical descent maneuver. Results also show that while a minimum $P_{S_{i}}$ magnitude can assure a minimum CPA for a given time-to-go, the same or greater CPA may be achievable with a lower magnitude of $P_{S_{i}}$ if the aircraft is able to gain sufficient speed to account for the loss of altitude (trade potential energy for kinetic energy).

Early evaluation made it clear that successful analysis would require compartmentalizing UAS performance capabilities into groups by UAS aerodynamic/propulsive performance, initial flight condition, commanded maneuver rate, and the encounter time to go. Three performance groups were developed for the descent maneuver: 1) the Sufficient Pitch Differential (SPD) group, which represents runs where the UAS is able to maintain the

17

American Institute of Aeronautics and Astronautics 
commanded descent rate throughout the encounter without gaining forward speed; 2) the Insufficient Pitch Differential (IPD) group, which contains runs where the UAS cannot maintain the commanded descent rate without accelerating; and 3) the Insufficient Time to Pitch (ITP) group, where the UAS is continually in the process of turning (pitching) to the commanded descent rate throughout the simulation. For the SPD group, a parametric equation was developed that can be used to predict the achievable CPA for a given UAS and encounter. The IPD group was more complex to characterize because the acceleration and maneuver behavior were more difficult to predict. The effects of various parameters were analyzed individually to identify which parameters have a large effect on the achievable CPA, and then, a method was introduced and demonstrated for deriving a series of performance parameter thresholds for a given encounter scenario and using them to predict SAA performance for a given UAS configuration. The ITP group was not analyzed in detail because this performance group contains a very small number of simulation runs and can be analyzed using simple geometric analysis. It is important to note that the performance groups are functions of the airplane configuration, encounter parameters, and the commanded maneuver rate; thus, a single airplane configuration can occupy any of the described performance groups.

The simulators, results, and methodologies presented in this paper attempted to establish a basis and framework for determining a minimum UAS performance that can be associated with performance of a given sense-and-avoid system. However, some of the specific analysis necessarily made assumptions about CPA ranges of interest as well as the overall design space.

Additionally, the simplified maneuvers and perfect sensors used in this study yield idealized results. Future studies will expand on the lessons learned from this work to study their more real-world implications associated with sensor noise and maneuver algorithms.

\section{Acknowledgments}

This work was supported by NASA's Unmanned Aircraft Systems Integration in the National Airspace System Project. In particular, support came from the Separation Assurance/Sense and Avoid Integration (SSI) element. The Co-Principal Engineer of the SSI Project, Maria Consiglio, at NASA Langley Research Center (LaRC) was the technical monitor for this project. Her efforts, difficult questions, and support ultimately made the work possible.

The work was supported through NASA LaRC Prime Contract NNL10AA14B: Task A089.001 to Stringer Ghaffarian Technologies (SGT) in Hampton, Virginia. Pierre Beaudoin was the project manager at SGT for the various UAS projects. Both he and SGT are a pleasure to work with.

Trevor Anderson, formerly of Adaptive Aerospace Group, did the early development of the simulator tool. His work, especially on the early GUI tool, helped shape the simulation.

The authors thank Peter Parker of NASA LaRC for helping with some of the numerical analysis and helping Devin learn his way around JMP ${ }^{\circledR}$.

Finally, feedback from James Chamberlain (LaRC) and people in the FAA and DoD, and members of RTCA SC-203 and 228 helped shape this work. The Proverb "iron sharpens iron" comes to mind.

\section{References}

1"General Operating and Flight Rules," 14 CFR Part 91, 2013.

${ }^{2}$ Federal Aviation Administration, "Sense and Avoid (SAA) for Unmanned Aircraft Systems (UAS) - Second Caucus Workshop Report," January 18, 2013.

${ }^{3}$ Federal Aviation Administration, "Introduction to TCAS II," version 7, November 2000.

${ }^{4}$ Jack, D. P., Hoffler, K. D., and Johnson, S. C., "Evaluation of the Trade Space Between UAS Maneuver Performance and SAA System Performance Requirements," NASA CR-2014-XXXXXX, May 2014.

${ }^{5}$ RTCA, Inc., "Terms of Reference, RTCA Special Committee 228, Minimum Performance Standards for Unmanned Aircraft Systems," RTCA Paper No. 109-13, May 20, 2013.

${ }^{6}$ RTCA, Inc., "Detect and Avoid (DAA) White Paper", RTCA Special Committee 228, Minimum Performance Standards for Unmanned Aircraft Systems, RTCA Paper No. SC228-WG1-WP01-01, November 1, 2013.

${ }^{7}$ SAS Institute Inc. JMP® 10 Discovering JMP. SAS Institute Inc., Cary, NC: 2012. 